\title{
Human Machine Integrated Interactions and Internet of Things
}

\author{
${ }^{1}$ Li Hua Fang and ${ }^{2}$ Dong Yonggui \\ 1,2 Institute of Opto-Electronic Engineering, Tsinghua University, Haidian District, Beijing, China, 100190. \\ 1lihua@tsinghua.edu.cn, ${ }^{2}$ dongyon332@hotmail.com
}

\begin{abstract}
ArticleInfo
Journal of Machine and Computing (http://anapub.co.ke/journals/jmc/jmc.html)

Doi : https://doi.org/10.53759/7669/jmc202101020

Received 23 March 2021; Revised form 30 June 2021; Accepted 12 August 2021; Available online 05 October 2021. (C)2021 The Authors. Published by AnaPub Publications.

This is an open access article under the CC BY-NC-ND license. (http://creativecommons.org/licenses/by-nc-nd/4.0/)

Abstract - More applications are being developed for the Internet of Things (IoT) these days. What resources are available to help with the software design phase, particularly for those programs that emphasize interplay? The incorporation of cognitive approach can aid in the understanding of people and the application of these discoveries in the design phase. The goal of this article is to introduce the idea of blending and the architecture of Integrated Interactions, and to apply those principles to the IoT. This article evaluates the intellectual foundations of conceptual integration and the layering process. After that, this article describes the Integrated Interaction framework and shows an Affinity Table in intervention. This paper also evaluates the fundamentals of the Internet of Things and how it can be used in various industries. According to the findings, this application offers a distinct blend.
\end{abstract}

Keywords - Internet of Things, Integrated Interaction, Human Computer Interaction, Human Machines, Computing Machines.

\section{INTRODUCTION}

Integrating the real and virtual worlds is critical in the internet of things regime. In light of the uncertainty and force of embedded systems, how could we design user-friendly functionalities or artifacts? This issue can only be considered by understanding how people gain knowledge and think. This perceptual context can then be applied to interaction design. The principle of "Integrated Interaction" will be discussed in detail in this article, as will the structure for it; the context of the internet of things will be described in this article, with respect to assumptions, which the theoretical frameworks can be advantageous in the context of internet of things. The phrase "embodied cognition" in behavioral sciences focuses on the significance of personal and cognitive interactions for human perception. Integration attempts to explain how much a person's body impacts their contextual cognitive, say academics e.g., speaking and thinking.

It is no secret that the way humans create sense of situations is heavily influenced by individual experience to a large extent. As a result, children's exploration of the world through physical interactions is critical for acquisition and the establishment of fundamental concepts. According to the authors, this not only means that people require a body to comprehend their surroundings, but also that thinking is founded on integration. Our conceptual frameworks and styles of reasoning are created by the same neurological and cognitive techniques that allow us to sense and move about. The following are 2 main breakthroughs in cognition:

- Studies of Human-Computer Interactions [1].

- Studies of Human-Computer Interactions in the internet of things regime [2].

Metaphors have both advantages and drawbacks. Although they can be misconstrued, the advantage of them is that they open up new ways of interacting depending on previous experience. They define literary metaphors as a conceptual tool rather than a poetic one for understanding. Actual psychological categorizations can be transferred to a new situation by using metaphors. A key part of the definition of the term is how it is used in context. "A domain is an area of activity that has been seen and characterized at an abstract level." Both abstract and concrete domains can exist in the same mind (maternal affection, the Titanic). More universal and less particular are the characteristics of conceptual realms. Defining one scope with the help of another is a common occurrence. To demonstrate the concept, researchers use the connotative "Love is indeed a Journey," whereby journey is the starting point and love is the destination [3].

Distinct projections take place as a result of this symbolism. Two lovers go on a trip together as companions to the same place. Once a map has been drawn, the model could be used in a more general sense. As an illustration, consider being forced to spend time with somebody. Analogies and other terminology can be viewed as guidelines for creating mental environments. When talking and thinking, conceptual spaces develop fragmented cognitive frameworks that "enable a fine-grained division of our dialogue and knowledge frameworks." They are classified as distinct domains and aid in environmental comprehension. Previous knowledge, cognition frameworks, observable acts, and discussions are all examples of sources for mental spaces, according to the researchers. Connectors can act as a bridge between distinct mental domains by establishing relationships between different characteristics of different places, culminating in a system 
of links. This paper has been organized as follows: Section II presents an analysis of the related works. Section III critically evaluates the theme while Section IV finally concludes the paper.

\section{RELATED WORKS}

P. Nyirenda, R. Bidarra and W. Bronsvoort in [4] presented a definition for a blend. To produce a blend, you combine two input areas into a third. The blend's structure depends on the two input spaces, but it also creates a new structure on its own. Based on a Buddhist monk's riddle, researchers explain how the blending process works. On his way up the hill, the monk takes time to contemplate before returning down the next morning. He does this every day. The objective is to find a place where the monk may be located at the same time of day on both travels. It is indeed easy to figure out by picturing the monk walking up and down the same street on the same day, and then running into somewhere along the way.

D. Stamenkovic in [5] argues that by approaching the riddle in this manner, the question of how to arrive at an answer and be certain that it is correct arises, even though such an interaction is not feasible. The answer lies in the way human sense-making functions. It makes no difference to human reasoning whether or not this situation can occur, as long as two people meet in fact. Human ingenuity makes it feasible to combine these two excursions into a single one, creating the possibility of meeting along the way.

K. Ghayur, R. Heaney and S. Platt in [6] argue that blending is a process composed of three phases. A cross-space mapping is used to link the relations and features of the two input domains at the start of the process (See Fig 1). The monk's two travels serve as input areas, as well as the cross-space mappings alludes to "mountainside, traveling people, day and movement."

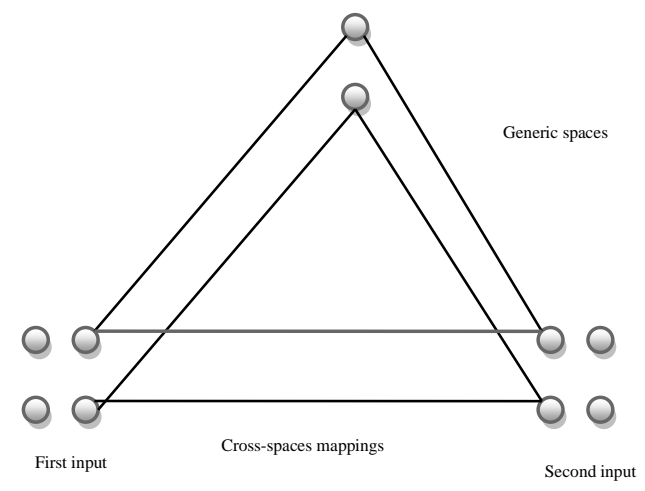

Fig 1. Cross-spaces mappings and generic spaces

Researchers in [7] argue that by expressing the common attributes of the cross-space mappings into one dimension (see Figure 1), a generalized space is formed. The general space of the puzzle has the following elements: "a moving person and his location, a road connecting the mountain's foot and its top, a day of journey, and motion in an undefined direction." In the end, the mix is created by partially projecting components from the two input areas (see Fig 2). The blend is nevertheless shaped by the input spaces, but it also has a new structure of its own. It's as though the combined space in the narrative of the monk encompasses both travels, complete with their own dynamics (different locations at various times of the day). Composition, completeness, and development are identified as three key aspects of blending by researchers. Composition is the process of establishing new connections between previously unconnected elements. In order to perceive blended structures as a single entity, completion allows the use of prior information. The mix can also be further refined, enlarged, and described by elaborating on it.

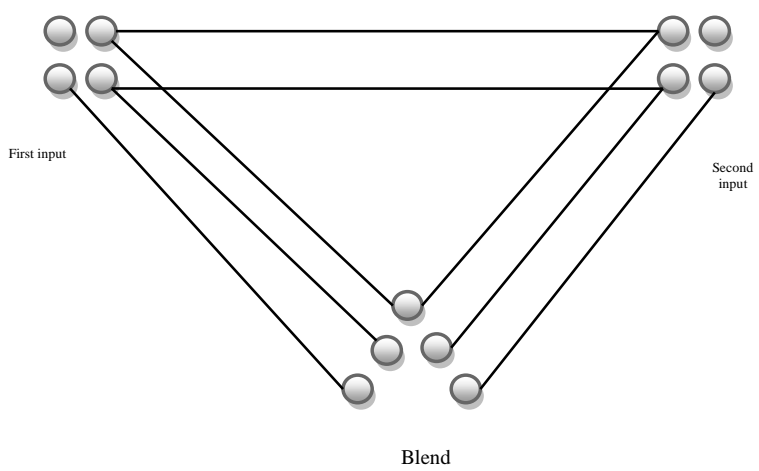

Fig 2. Integrated (Blend) spaces

Researchers in [8] conducted an analysis of the desktop metaphor regarded as a blend. A blend is created when office supplies like paper and pencils are combined with computer codes like cut / paste. The desktop metaphor has its roots in the Xerox Star and the goal of expanding on well-known ideas to make computers easier to comprehend. It has developed 
into a vast mix since its inception as a desktop interface and continues to do so now. Desktop users are well-versed in the fact that, for example, a window (along with other things) on the screen is not the same as windows in real life. Most typical office chores are still kept as activities in metaphor, which means metaphor is a phrase that refers to desktop interfaces (for example, opening and closing of folders).

I. Bouyukliev, V. Fack, W. Willems and J. Winne in [9] argue that the operations that are carried out mirror those of an office work, and little attention needs to be paid to the corresponding computer codes. A desktop computer represents the operating system in the analogy used. Nevertheless, on closer inspection, it's clear that the desktops experience is a synthesis based on the office paradigm. There are actions, such as opening a folder with a double click, that do not exist in any of the two input areas, giving the desktops interaction a complex feature. There are two sorts of mixing principles, according to the research. Constitutive concepts are the basic concepts that characterize an activity and distinguish it from other activities by setting regulations. Section III presents a critical analysis of integrated interactions.

\section{CRITICAL ANALYSIS}

The Internet of Things is about connecting physical items to the Internet. Thus, the Internet of Things (IoT) promises to bridge the physical and digital divide by integrating computing elements into a wide range of everyday activities, places, and objects. In other words, the Internet of Things opens up a whole new world of interface design possibilities. We have already seen some great instances of how this is beginning to work in practice. The number of networked items, such as connected automobiles, specialized computers, and tagged objects, is fast increasing as well.

Industry and academics both have a vested interest in the Internet of Things. While the technology progress in this field is exciting, we find it much more intriguing to watch where interface design will go in the coming years. From the standpoint of interface design, it is always exciting to see what new user experiences and digital services may be developed using this digital content. For us, the Internet of Things (IoT) is both an application field and a new design material, therefore we want to approach it from a material-centered perspective. Because of this approach, we aim to move beyond the services that internet-enabled devices may give and instead focus on re-imagining what human interaction may be used for, i.e. how IoT could extend the design scope of HCI design.

In the internet of things, anything that can be given an IP addresses and send data across a network is included. Examples include people with implanted heart monitors, agricultural animals with biochip transponders, and automobiles with tire pressure monitoring systems. Organizations in a wide range of sectors are increasingly relying on the Internet of Things (IoT) to run more effectively, better understand their consumers, and provide better customer service while also improving decision-making and the company' value. Embedded systems including CPUs, sensors, and communication hardware make up an Internet of Things (IoT) ecosystem because they enable web-enabled smart objects to gather data from their surroundings, communicate it, and take action on it. By connecting with an Internet of Things gateway or other edge device, sensor data may be shared and analyzed in the cloud or on-site.

These gadgets are capable of exchanging information and taking action in response to it. Despite the fact that people may use the devices to set them up, give them instructions, or retrieve data, the devices do the majority of the work without human involvement. People can live and work more intelligently and completely manage their life thanks to the Internet of Things. IoT is critical to businesses as well as homeowners in terms of smart home automation. It gives organizations a glimpse into how their systems truly operate, giving them information on anything from equipment performance to supply chain and logistical processes. Automation and labor cost reduction are both made possible by the Internet of Things (IoT). It also reduces waste and enhances service delivery, resulting in lower manufacturing and delivery costs and greater transparency for customers in their interactions. Thus, the Internet of Things (IoT) is a critical technology for everyday living, and it will continue to gain traction as more companies see the value in using smart devices to maintain a competitive advantage.

\section{Integrating Principles and Techniques}

There are three basic principles: synthesis, conclusion, and development. In contrast to constitutive principles, which provide rules for the blending process and cognitive spaces, regulatory concepts (or ethical guidelines) convey techniques for improving the progression of blending. Both sorts of principles have limits in that they indicate what will not happen, but they cannot be utilized to forecast the end result. Developing blends at a degree of abstraction that people can comprehend and focus on the essential aspects without any unnecessary details is a crucial component of applying these concepts for blending [10]. Distinct systems of government have different ideals. While it is not always feasible to meet all of these requirements at once, keeping them in mind might help when making design selections. A disagreement between two principles necessitates the identification of the most critical notion. The following sections summarize several mixing concepts based on researchers' results.

\section{Comprehension}

A blend is a condensed representation of the relationships between the multiple input areas. To make the mix more humanfriendly, focus on characteristics of the space that are easier for humans to grasp. Only the most relevant parts and states should be audible during the blending process. The trash can symbol, for instance, has two possible states in the desktops metaphors. It can be empty or full, depending on how many elements are included within it. There aren't any intermediate stages for varying the amount of items because they aren't required in a desktop setting. 


\section{Topology}

The topology explains the arrangement of things. You can choose between inner- and outer-space topologies. The innerspace is concerned with interconnections inside a single input space, while the outer-space is concerned with relationships between various input spaces. To the mix, a number of different projection topologies can be used. Because the trash can is generally found below the desk while using the desktop interface, it's easy for users to lose track of where it belongs.

\section{Integration}

The integration process is used by human brains. There are many different methods of integration, but they all revolve around the development of a mix that is viewed as a single unit. It's important that the mix is seen as consistent rather than as something that's been put together from various components. Humans will find it much easier to use and remember if it is designed this way. Pattern fulfillment, networking, and decompression are all ways to achieve integration. The methodical use of existing background information to complete the blend, for example, utilizing the office frame of the desktops metaphors in the setting of some other metaphor to provide extra functionalities, is known as pattern completion. The Web is concerned with making forward linkages from the mix return to the initial input subdomains. Regardless of the fact that focus is on the mix and not the initial input regions, human beings should be able to feel these linkages to help comprehension. A key concept of mix deconstruction is that it should be clear how the blend was put together. The availability of suitable links between input areas is a critical factor in streamlining this procedure.

\section{Recursion and Relevance}

Unnecessary ingredients should not be used in a mix. You should get rid of anything that is unrelated. The idea of recursion refers to the potential of repeating a blend as an entry for yet another blending and so achieving a reduction level. Studies reported how a blending is produced between an input method like the mouse and a graphical interface using the instance of the subfolder blending (see Fig 3). This is an example of blending two different sources. To begin with, a manipulation blend is generated by combining the mouse's input space with the manual action's input area. As a result, there are now three more actions in the mix.

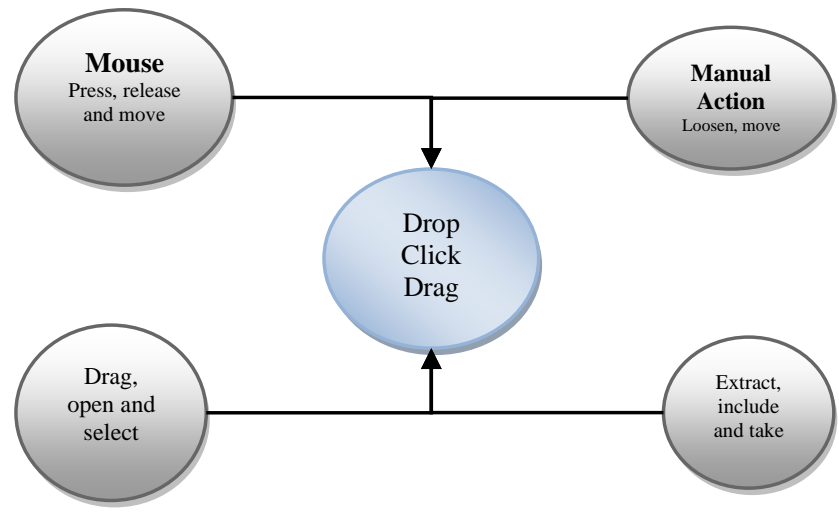

Fig 3. The blend's forder

A click is a selecting tool that involves pushing and releasing the mouse button. The open action is represented by two clicks, and a double click is connected with each one. To move an object, simply hold down the mouse button and drag it in the direction you want it to go. When the mouse button is released, a drop is performed to put an end to the dragging motion.

The manipulating blend is coupled with a physical folder to generate the folder blend using the governing concept of recursion, which states that a blend can be used as input for another blend. The folder blend yields the folder icon and numerous operations that can be applied to its digital representation. A folder may be opened by double-clicking its icon once it has been selected with a single click. Additionally, digital folders may be dragged and dropped and items can be added to the folder by dragging and dropping them there.

\section{Integrated Interactions}

This theory of "Integrated Interaction" was made by scientists in terms of explaining why functionality is regarded as "organic" or not. Simple and intuitive interface can be intended for living beings by taking into account the intended surroundings and developing specific suggestions that take this into account. The platform is intended as an explanation tool for post-"Windows Symbols Menu Pointer" collaboration settings. In the following section, we shall show how blending may be beneficial in HCI while also outlining the framework for Integrated Interaction and its elements.

\section{Integrating in Human-Computer Interactions}

Experts define human-computer interaction as a mix based on the PACT paradigm, which argues that human-computer interaction has four major components. This framework focuses on the following four essential elements: people; activities; situations; and technology (see figure 4). It is a goal to combine those four components. 


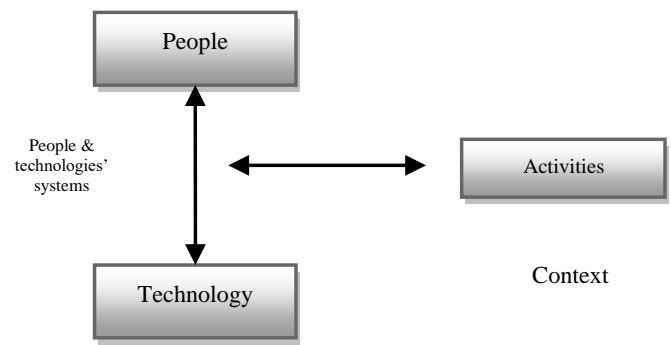

Fig 4: Technologies, contexts, activities and people framework

Human Computer Interaction (HCI) blends individuals, actions, and surroundings with computers. In contrast to technological activities, human activities strive to achieve human goals and hence do not require instruction as a set of acts. Compressing the tension between these two characteristics is a significant challenge in HCI research. According to their findings, the perceptions we have in the physical world are distinct from those we have in the virtual environment. The designer's job is to create a seamless transition between the real and virtual realms while maintaining the knowledge and computational capability of each. Because they share certain characteristics of the actual world, user interfaces don't have to be created to be as accurate as feasible. When designing interfaces, it's possible that the designers' and users' mental spaces diverge, which presents a challenge.

This means that interaction should be built on a generic area that most people share, such as picture schema, study interpretations of capabilities, and the four realities. This section goes into further detail about each of those three ideas. To be intuitive in communication, you must be able to use previously acquired knowledge without being aware of it. Instinctual or multisensory knowledge are both examples of which was before knowledge. Genes or prenatal experiences specify innate knowledge. Somatosensory knowledge, which comprises conceptual frameworks and attributes of pictures, is acquired in early life. Abstract components that people employ to think about the surroundings are represented by image schemata. They're made up of patterns that emerge regularly in bodily encounters. Consider the container schema, which consists of an inner and outer portion that are divided by a border. According to this model, an hour glass is made up of two containers, one filled with a material and the other linked by a link.

\section{Internet of Things and Integrated Interactions}

IoT may be described as a broad term that has several different subsets inside it. Smart products and apps typically combine elements from the physical and digital worlds.. This section defines "Internet of Things," "smart things," and provides examples of its use in many industries. In addition, we plan to apply the principles of Blended Interaction and Blended Blending to the IoT (see Fig 5).

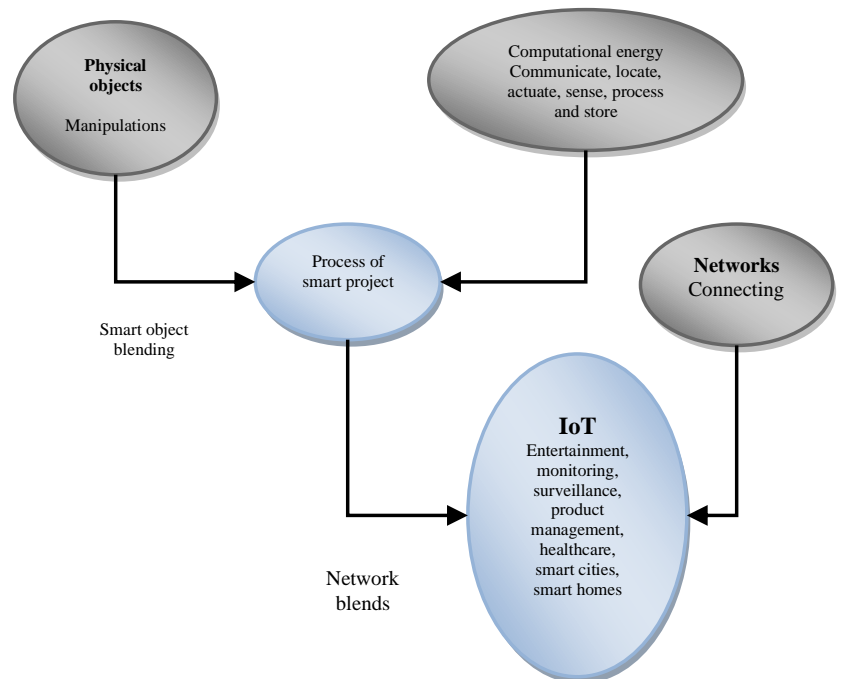

Fig 5. IoT considered a blend

\section{Theoretical Basis of IoT}

Internet of things is a difficult concept to define. A wide range of movements are incorporated into this dance style. Their origins can be traced back to the Ubiquitous Computing movement. It is based on the concept of seamless computer interconnection into the surroundings that researchers have initiated as Pervasive Computing. Computer-generated realism differs from virtual reality in that instead of creating an entirely new world, the computer just inserts itself into the existing one. The goal does not end with the portability of desktop computers. As a result, new forms of interaction are possible without being constrained by the gadget. The concept of Ambient Intelligence, which is founded on the concept of Ubiquitous Computing, is similar to that of the Internet of Things. 
Algorithmically enhancing the environment with a focus on human needs and well-being is at the heart of Ambient Intelligence's mission. According to experts, the Internet of Things idea is founded on the belief that advances in microelectronic devices, telecommunication, and information systems will keep rising. Technology is becoming increasingly integrated into items as a result, creating new opportunities for engagement. Things that are "smart" are capable of interacting with one another, as well as with people. The phrase "Internet of Things" refers to both a network of internet-connected smart items and the enabling technologies that will make this vision a reality. The following technical advances, according to W. Wang in [11], allow for a tighter connection between the real and virtual world and can be viewed as smart object features.

- Cooperation and communication - objects are linked with the web or with one another and could assess datasets

- Identification and addressability - each object can potentially be addressing within a particular network and can be identified uniquely.

- Sensing - different sensors integrated within different objects allow data collected on the surrounding. This form of data is saved, transffered or processed.

- Actuation - Actuators incorporated within objects affect the environment (for instance changing electronic inputs unto motion.

- Embedded data processing - objects integrated microprocessors and the capability to store information.

- Localization - smart objects are informed about their positions within the surrounding or locations could be identified.

- Users' interface - smart objects have both an indirect and direct interface through hich users can manipulate them and enhance accessibility to data. For example, indirect interactions could happen through mobile phones.

The Internet of Things (IoT) may be utilized in smart buildings or smart houses to reduce energy usage (such as water use) or improve the quality of life for the occupants. Energy usage in the building may be monitored via sensors, and occupants' requirements can be identified and met with appropriate responses (such as switching on/off). The field of smart buildings and mobility is concerned with the effective management of the city's infrastructure. Improved traffic management may be aided by new technologies. The presence of automobiles in the municipal surveillance system The technology may make it easier to avoid traffic jams and catch traffic offenses.

Furthermore, parking space and pollution detection may be available. Environmental monitoring is used to identify natural events such as rainfall or temperature changes. The main problem in this field is to integrate a large number of sensors while also analyzing and processing the recorded data in real time. It is possible to monitor the ecosystem in locations that are inaccessible to people, such as the ocean or a volcanic cone. In the event of an emergency or a catastrophe, these devices can alert people before it is too late. When used in healthcare system, the IoT can help with assisted living. Patients may be monitored from home without having to visit a hospital, and their medical information can be sent to specialists across the country or even the world. As a result, critical situations are more likely to be recognized in time. Another component of health that has to do with self-tracking is exercise. People may now keep track of their personal workouts and activities with the help of this app. Smart gadgets can provide suggestions for ways to improve health based on the data they collect from users. Inventory and corporate development systems for tracking and identifying items are in use in smart companies.

It is a start, but there is more to be done. The employment of bio-sensors and technologies to increase the flexibility of procedures can assist provide data on the status of items (for example temperature or appearance of bacteria). The Internet of Things (IoT) has the potential to assist improve security and surveillance operations. Analyzing the surroundings might help identify persons who are behaving inappropriately. Devices might also be made to be less conspicuous and less annoying. The music industry can facilitate the delivery of user-specific news about current media equipment based on their location. In addition to serving as a medium of communication, placing tags on locations can assist convey extra data to the receiver.

\section{CONCLUSION}

Blending and Integrated Interactions ideas, as well as their cognitive roots, have been discussed in depth in this study. We agree with the research that says that while designing interfaces, it is critical to keep in mind how humans build mental representations and reasoning about the environment. If such interfaces are well-integrated, people will have an easier time picking them up faster than usual. We discovered that the concepts of blending and the architecture Integrated Interactions came in handy. We can see how including this framework into the design process may be beneficial for the creation of smart products in the future. It is important to remember that the Integrated Interaction architecture is designed for cooperative engagement. Even if the design domain of communication and social interaction is ignored, we believe it would be advantageous to consider personal interactions in the system design.

\section{References}

[1]. J. Long, "Specifying relations between research and the design of human-computer interactions", International Journal of Human-Computer Studies, vol. 44, no. 6, pp. 875-920, 1996. Doi: 10.1006/ijhc.1996.0037.

[2]. P. Chhikara, P. Singh, R. Tekchandani, N. Kumar and M. Guizani, "Federated Learning Meets Human Emotions: A Decentralized Framework for Human-Computer Interaction for IoT Applications", IEEE Internet of Things Journal, vol. 8, no. 8, pp. 6949-6962, 2021. Doi: 10.1109/jiot.2020.3037207.

[3]. J. Fins, "In an Instant: A Family's Journey of Love and Healing", JAMA, vol. 297, no. 23, p. 2641, 2007. Doi: 10.1001/jama.297.23.2642.

[4]. P. Nyirenda, R. Bidarra and W. Bronsvoort, "A Semantic Blend Feature Definition", Computer-Aided Design and Applications, vol. 4, no. 6, pp. 795-806, 2007. Doi: 10.1080/16864360.2007.10738512. 
[5]. D. Stamenkovic, "Animated Visual Stimuli in Blending: Solving the Riddle of the Buddhist Monk (Status Report, September 2014)", SSRN Electronic Journal, 2014. Doi: 10.2139/ssrn.2496010.

[6]. K. Ghayur, R. Heaney and S. Platt, "Constructing Long-Only Multifactor Strategies: Portfolio Blending vs. Signal Blending", Financial Analysts Journal, vol. 74, no. 3, pp. 70-85, 2018. Doi: 10.2469/faj.v74.n3.5.

[7]. H. Anandakumar, R. Arulmurugan, and A. Roshini, "Intelligent Vehicle System Problems and Future Impacts for Transport Guidelines," 2019 International Conference on Smart Systems and Inventive Technology (ICSSIT), Nov. 2019

[8]. E. Pimenidis, D. Williams and A. Pollard, "Thinking about Android Epistemology * Beyond the Desktop Metaphor Designing Integrated Digital Work Environments * Business Knowledge for IT in Retail Banking", ITNOW, vol. 50, no. 2, pp. 27-27, 2008. Doi 10.1093/itnow/bwn039.

[9]. I. Bouyukliev, V. Fack, W. Willems and J. Winne, "Projective two-weight codes with small parameters and their corresponding graphs", Designs, Codes and Cryptography, vol. 41, no. 1, pp. 59-78, 2006. Doi: 10.1007/s10623-006-0019-1.

[10]. V. Bermejo and J. Díaz, "The Degree of Abstraction in Solving Addition and Subtraction Problems", The Spanish Journal of Psychology, vol. 10, no. 2, pp. 285-293, 2007. Doi: 10.1017/s1138741600006557.

[11]. W. Wang, "Difference between the Real World and Virtual World", Proceedings, vol. 47, no. 1, p. 35, 2020. Doi: $10.3390 /$ proceedings 202004703 